\title{
Kontaktbedingte Veränderung der Hilfsverbselektion im Cimbro : Ergebnisse einer Pilotstudie
}

Kolmer, Agnes

Posted at the Zurich Open Repository and Archive, University of Zurich

ZORA URL: https://doi.org/10.5167/uzh-40651

Book Section

Published Version

Originally published at:

Kolmer, Agnes (2010). Kontaktbedingte Veränderung der Hilfsverbselektion im Cimbro : Ergebnisse einer Pilotstudie. In: Scherer, Carmen; Holler, Anke. Strategien der Integration und Isolation nicht-nativer Einheiten und Strukturen. Berlin: De Gruyter, 143-164. 
Germanistik Online Datenbank

Internationales Referatenorgan mit bibliographischen Hinweisen 1 (1960) - 57 (2016)

Hrsg. v. Bastert, Bernd / Jacob, Joachim / Kämper, Heidrun / Kilian, Jörg / Kimmich, Dorothee / Kragl, Florian / Lubkoll, Christine / Schneider, Sabine

Erschienen im Druck: 2012-90

Publikationstyp: Aufsatz

Autor: Kolmer, Agnes

Titel: Kontaktbedingte Veränderung der Hilfsverbselektion im Cimbro. Ergebnisse einer Pilotstudie

Details / Quelle: Strategien der Integration und Isolation nicht-nativer Einheiten und Strukturen

Erscheinungsjahr: 2010

Umfang: $143-164$

Schlagwort(e): Italien (Deutsch)Luserna/Italien (Deutsch)Lexikologie (Fremdwort)ZimbrischSprachinselSprachkontaktDeutsch/ItalienischFremdwortLehnbeziehung Klassifikation: IV. Allgemeine und indogermanische Sprachwissenschaft / Kontrastive Sprachwissenschaft

Beitrag aus:

- Strategien der Integration und Isolation nicht-nativer Einheiten und Strukturen

Zugang bereitgestellt von: UZH Hauptbibliothek / Zentralbibliothek Zürich

Copyright $\odot$ 2011-2017 by Walter de Gruyter GmbH

Powered by PubFactory 